\title{
On Construction of Mean Graphs
}

\author{
P. Jeyanthi ${ }^{1 *}$ and R. Ramya ${ }^{2}$ \\ ${ }^{1}$ Research Centre, Department of Mathematics, Govindammal Aditanar College for Women, \\ Tiruchendur-628 215, India \\ ${ }^{2}$ Department of Mathematics, Dr.Sivanthi Aditanar College of Engineering, Tiruchendur-628 215, \\ India
}

Received 6 August 2012, accepted in final revised form 26 February 2013

\begin{abstract}
A graph $G=(p, q)$ with $p$ vertices and $q$ edges is called a mean graph if there is an injective function $f$ that maps $V(G)$ to $\{0,1,2,3, \ldots, q\}$ such that for each edge $u v$, is labeled with $\frac{f(u)+f(v)}{2}$ if $f(u)+f(v)$ is even and $\frac{f(u)+f(v)+1}{2}$ if $f(u)+f(v)$ is odd. Then the resulting edge labels are distinct. In this paper, we prove some general theorems on mean graphs and show that the graphs $G=P_{m}(+) \overline{K_{n}}$, Jewel graph $J_{n}$, Jelly fish graph $(J F)_{n}$ and $K_{n}^{c}+2 P_{3}$ are mean graphs.

Keywords: Mean labeling; Mean graph. (C) 2013 JSR Publications. ISSN: 2070-0237 (Print); 2070-0245 (Online). All rights reserved. doi: http://dx.doi.org/10.3329/jsr.v5i2.11545 J. Sci. Res. 5 (2), 265-273 (2013)
\end{abstract}

\section{Introduction}

By a graph we mean a finite, simple and undirected one. The vertex set and the edge set of a graph $G$ are denoted by $V(G)$ and $E(G)$ respectively. The disjoint union of $m$ copies of the graph $G$ is denoted by $m G$. The union of two graphs $G_{1}$ and $G_{2}$ is the graph $G_{1} \cup G_{2}$ with $V\left(G_{1} \cup G_{2}\right)=V\left(G_{1}\right) \cup V\left(G_{2}\right)$ and $E\left(G_{1} \cup G_{2}\right)=E\left(G_{1}\right) \cup E\left(G_{2}\right)$. A vertex of degree one is called a pendant vertex. Let $G=(p, q)$ be a mean graph with $p$ vertices and $q$ edges and let $v$ be a vertex with label $q$ and let one of the mean labelings of $G$ satisfy the following: If $q$ is odd (even) and all the labels of the vertices which are adjacent to $v$ are even (odd), then we call this mean labeling as extra mean labeling [4] and the graph $G$ as extra mean graph.

\footnotetext{
*Corresponding author: jeyajeyanthi@rediffmail.com
} 
The Jewel graph $J_{n}$ is a graph with vertex set $V\left(J_{n}\right)=\left\{u, x, v, y, u_{i}: 1 \leq i \leq n\right\}$ and edge set $E\left(J_{n}\right)=\left\{u x, v x, u y, v y, x y, u u_{i}, v u_{i}: 1 \leq i \leq n\right\}$. The graph Jelly fish $(J F)_{n}$ has $2 n$ vertices and $2 n+1$ edges with vertex set $V\left((J F)_{n}\right)=\left\{u, v, u_{i}, v_{j}: 1 \leq i \leq n, 1 \leq j \leq n-2\right\}$ and edge set $E\left((J F)_{n}\right)=\left\{u u_{i}: 1 \leq i \leq n\right\} \cup\left\{v v_{i}: 1 \leq i \leq n-2\right\} \cup\left\{u_{1} u_{n}, v u_{1} v u_{n}\right\}$. Terms and notations not defined here are used in the sense of Harary [1].

The concept of mean labeling was introduced by Somasundaram and Ponraj [2] and further studied by the same authors in [3]. Motivated by the work of the above authors, we have established the mean labeling of some standard graphs in [4,5]. In this paper we extend our study to establish the mean labeling some more graphs like Jewel graph $J_{n}$ and Jelly fish graph $(J F)_{n}$.

\section{Mean Graphs}

Remark 2.1: For any mean graph $G, 0, q-1$ and $q$ must be the vertex labels. Either 1 or 2 must be a vertex labeling, a vertex of label $q-1$ is adjacent with a vertex of label $q$ and a vertex of label 0 is adjacent with a vertex of label 1 or 2 .

Theorem 2. 2: Let $G_{1}=\left(p_{1}, q_{1}\right)$ be a mean graph with mean labeling $f$ and let $e=x u$ be an edge with $f(x)=q_{1}-1$ and $f(u)=q_{1}$. Let $G_{2}=\left(p_{2}, q_{2}\right)$ be a mean graph with mean labeling $\mathrm{g}$ and let $e^{\prime}=y v$ be an edge with $g(y)=0$ and $g(v)=1$ (or 2). If $G$ is a graph obtained by joining the vertex $x$ with $y$ and $u$ with $v$ by an edge, then $G$ is a mean graph.

Proof: Add the number $q_{1}+2$ to all the vertex labels of the graph $G_{2}$.Then the vertex labels of $G_{2}$ remain distinct and the edge labels of $G_{2}$ are increased by $q_{1}+2$. That is the edge labels of $G_{2}$ are $q_{1}+3, q_{1}+4, \ldots, q_{1}+q_{2}+2$. Now the label of the edge $x y$ is $\left\lceil\frac{q_{1}-1+q_{1}+2}{2}\right\rceil=\left\lceil\frac{2 q_{1}+1}{2}\right\rceil=q_{1}+1$. Also the label of the edge $u v$ is $\left\lceil\frac{q_{1}+q_{1}+3}{2}\right\rceil=q_{1}+2$ if $g(v)=1$ and the label of the edge $u v$ is $\left\lceil\frac{q_{1}+q_{1}+4}{2}\right\rceil=q_{1}+2$ if $g(v)=2$. Hence the edge labels of the graph $G$ are $1,2,3, \ldots, q_{1}+q_{2}+2$ and the vertex labels of $G$ are also distinct. This completes the proof.

Example 2.3: Let $G_{1}=P_{4}$ and $G_{2}=S\left(K_{1,3}\right)$. The mean labeling of $G_{1}$ and $G_{2}$ are given below.

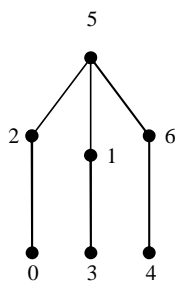


The mean graph obtained by the above construction is given in Fig. 1.

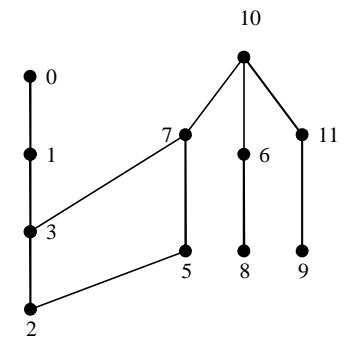

Fig. 1

Theorem 2.4: Let $G_{1}=\left(p_{1}, q_{1}\right)$ be a mean graph with mean labeling $f$ and let $e=u x$ be an edge with $f(x)=q_{1}-1$ and $f(u)=q_{1}$ and let $G_{2}=\left(p_{2}, q_{2}\right)$ be a mean graph with mean labeling $g$ and let $e^{\prime}=v y$ be an edge with $g(y)=0$ and $g(v)=1$. If $G$ is a graph obtained by identifying the edge $e^{\prime}$ with the edge $e$ (that is identifying $u$ with $v$ and $x$ with $y$ ), then $G$ is a mean graph.

Proof: Let $V\left(G_{1}\right)=\left\{u, x, u_{i}: 1 \leq i \leq p_{1}-2\right\}$ and $V\left(G_{2}\right)=\left\{v, y, v_{i}: 1 \leq i \leq p_{2}-2\right\}$. Then $V(G)=\left\{u=v, x=y, u_{i}, v_{j}: 1 \leq i \leq p_{1}-2,1 \leq j \leq p_{2}-2\right\}$. Clearly $G$ has $p_{1}+p_{2}-2$ vertices and $q_{1}+q_{2}-1$ edges.

$$
\text { Define } h: V(G) \rightarrow\left\{0,1,2,3, \ldots, q_{1}+q_{2}-1\right\} \text { by } h(w)=\left\{\begin{array}{lll}
f(w) & \text { if } & w \in V\left(G_{1}\right) \\
g(w)+q_{1}-1 & \text { if } & w \in V\left(G_{2}\right)
\end{array}\right. \text {. }
$$

Here $h(u)=h(v)=q_{1}$ and $h(x)=h(y)=q_{1}-1$. Since $G_{1}$ and $G_{2}$ are mean graphs and the vertex labels of $G_{2}$ are increased by $q_{1}-1$, the vertex labels of $G$ are distinct. The edge labels of the graph $G_{1}$ under $h$ are $1,2,3, \ldots, q_{1}$ and the edge labels of $G_{2}$ (except $e^{\prime}$ ) under $h$ are $q_{1}+1, q_{1}+2, \ldots, q_{1}+q_{2}-1$. Hence $G$ is a mean graph.

Example 2.5: Let $G_{1}=C_{5}$ and $G_{2}=P_{4}$. The mean labeling of $G_{1}$ and $G_{2}$ are given below.
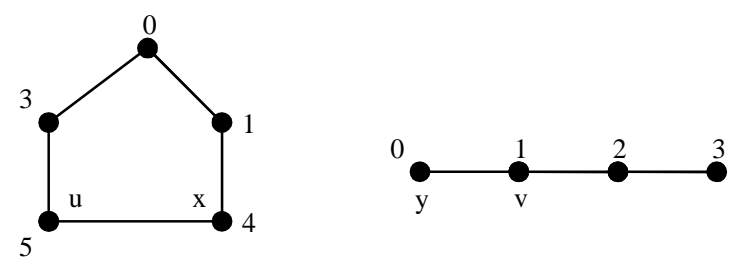

The mean graph obtained by the above construction is given Fig. 2. 


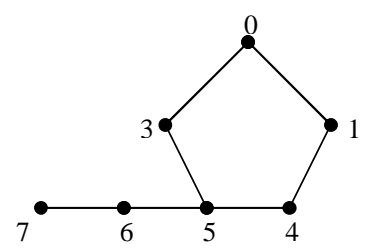

Fig. 2

Theorem 2.6: Let $G_{1}=\left(p_{1}, q_{1}\right)$ be an extra mean graph with an extra mean labeling $f$ and let $e=x u$ be an edge with $f(x)=q_{1}-1$ and $f(u)=q_{1}$. Let $G_{2}=\left(p_{2}, q_{2}\right)$ be a mean graph with mean labeling $g$ and let $e^{\prime}=y v$ be an edge with $g(y)=0$ and $g(v)=2$. The graph $G$ obtained by identifying the edge $e^{\prime}$ with the edge $e$ (that is identifying $x$ with $y$ and $u$ with $v$ ), then $G$ is a mean graph.

Proof: Let $V\left(G_{1}\right)=\left\{u, x, u_{i}: 1 \leq i \leq p_{1}-2\right\} \quad$ and $V\left(G_{2}\right)=\left\{v, y, v_{i}: 1 \leq i \leq p_{2}-2\right\}$. Then $V(G)=\left\{u=v, x=y, u_{i}, v_{j}: 1 \leq i \leq p_{1}-2,1 \leq j \leq p_{2}-2\right\}$. Clearly $G$ has $p_{1}+p_{2}-2$ vertices and $q_{1}+q_{2}-1$ edges.

Define $h: V(G) \rightarrow\left\{0,1,2,3 \ldots, q_{1}+q_{2}-1\right\}$ by $h(u)=q_{1}+1 ; h(x)=q_{1}-1$; $h\left(u_{i}\right)=f\left(u_{i}\right)$ for $1 \leq i \leq p_{1}-2$ and $h\left(v_{j}\right)=g\left(v_{j}\right)+q_{1}-1$ for $1 \leq j \leq p_{2}-2$.

Since $G_{1}$ is a mean graph, the vertex labels of $G_{1}$ under $h$ are remain distinct and $h\left(V\left(G_{1}\right)\right) \subseteq\left\{0,1,2, \ldots, q_{1}-1, q_{1}+1\right\}$. Since the label of the vertices of $V\left(G_{2}\right)-\{y, v\}$ are increased by $q_{1}-1$ and $G_{2}$ is a mean graph, the labels of the vertices of $V\left(G_{2}\right)-\{y, v\}$ are distinct. Also $h\left(V\left(G_{2}\right)-\{y, v\}\right) \subseteq\left\{q_{1}, q_{1}+2, \ldots, q_{1}+q_{2}-1\right\}$. The edge labels of the graph $G_{1}$, except the edges incident with $u$, under $h$ remain distinct. Since $G_{1}$ is an extra mean graph with mean labeling $f$, for each vertex $w$ incident with $u$ in $G_{1}, f(u)$ and $f(w)$ are of opposite parity. Therefore the induced edge label under $f$ is

$$
\begin{aligned}
& f *(u w)=\left\lceil\frac{f(u)+f(w)}{2}\right\rceil=\frac{q_{1}+f(w)+1}{2}=k, \text { an integer. Also, } \\
& h^{*}(u w)=\left\lceil\frac{h(u)+h(w)}{2}\right\rceil=\frac{q_{1}+1+f(w)}{2}=k .
\end{aligned}
$$

Hence, the induced edge labels of $G_{1}$ under $h$ are $1,2,3, \ldots, q_{1}$ and the edge labels of $G_{2}$ (except $e^{\prime}$ ) under $h$ are $q_{1}+1, q_{1}+2, \ldots q_{1}+q_{2}-1$. Hence $G$ is a mean graph.

Example 2.7: Let $G_{1}=C_{6}$ and $G_{2}=C_{6}$. The extra mean labeling of $G_{1}$ and a mean labeling of $G_{2}$ are given below. 


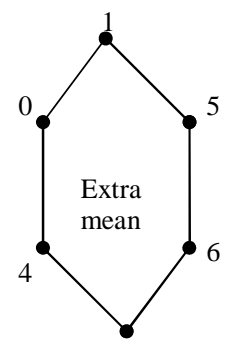

3

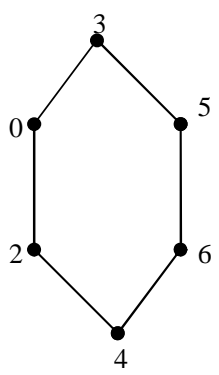

The mean graph obtained by the above construction is given in Fig. 3 .

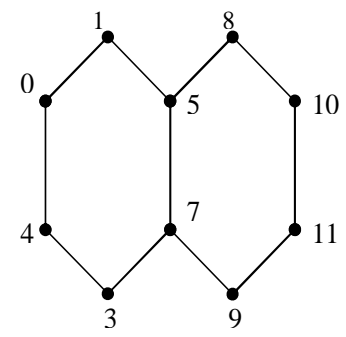

Fig. 3

Theorem 2.8: The Jewel graph $J_{n}$ is an extra mean graph.

Proof: Let $V\left(J_{n}\right)=\left\{u, x, v, y, u_{i}: 1 \leq i \leq n\right\}$ and $E\left(J_{n}\right)=\left\{u x, v x, u y, v y, x y, u u_{i}, v u_{i}: 1 \leq i \leq n\right\}$. Then $J_{n}$ has $n+4$ vertices and $2 n+5$ edges. Define $f: V\left(J_{n}\right) \rightarrow\{0,1,2, \ldots, 2 n+5\}$ as follows:

$$
f(u)=0 ; f(v)=2 n+5 ; f(x)=2 ; f(y)=2 n+4 ; f\left(u_{i}\right)=2 i+2 \text { for } 1 \leq i \leq n .
$$

For each vertex label $f$, the induced edge label $f *$ is defined as follows:

$$
\begin{aligned}
& f *\left(u u_{i}\right)=i+1 \text { for } 1 \leq i \leq n ; f *\left(v u_{i}\right)=n+i+4 \text { for } 1 \leq i \leq n \\
& f *(u x)=1 ; f *(u y)=n+2 ; f *(x v)=n+4 ; f *(v y)=2 n+5 \\
& f *(x y)=n+3
\end{aligned}
$$

Clearly $\mathrm{f}$ is a mean labeling of $G$. Moreover $q$ is odd and all the vertices which are adjacent to the vertex labeled $q$ are even. Thus, $G$ is an extra mean graph.

Example 2.9: The mean labeling of $J_{5}$ is given in Fig. 4 . 


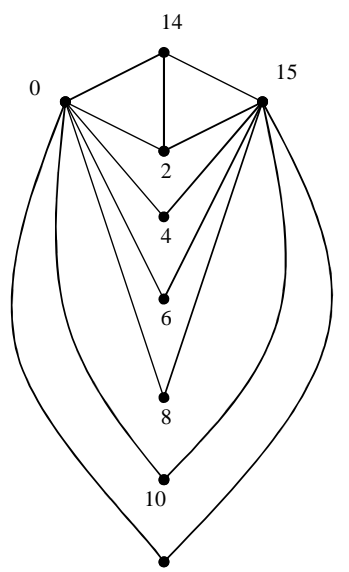

12

Fig. 4

Theorem 2.10: Let $G=P_{m}(+) \overline{K_{n}}$ be the graph with the vertex set $V(G)=\left\{u_{i}, v_{j}: 1 \leq i \leq m, 1 \leq j \leq n\right\}$ and the edge set $E(G)=\left\{u_{i} u_{i+1}, u_{1} v_{j}, u_{m} v_{j}: 1 \leq i \leq m-1\right.$ and $\left.1 \leq j \leq n\right\}$. Then $G$ is a mean graph.

Proof: Let $V(G)=\left\{u_{i}, v_{j}: 1 \leq i \leq m, 1 \leq j \leq n\right\}$.

Define $f: V(G) \rightarrow\{0,1,2, \ldots, m+2 n-1\}$ as follows:

$$
f\left(u_{1}\right)=0, \begin{array}{ll}
2 n+2 i-3 & \text { for } \quad 2 \leq i \leq\left\lceil\frac{m+1}{2}\right\rceil \\
2 n+2+2(m-i) & \text { for } \quad\left\lceil\frac{m+1}{2}\right\rceil+1 \leq i \leq m
\end{array} \text { and }
$$

$f\left(v_{j}\right)=2 j \quad$ for $1 \leq j \leq n$. Then $f(V(G))=\{0,2,4, \ldots, 2 n, 2 n+1,2 n+2, \ldots, 2 n+m-1\}$.

For each vertex label $f$, the induced edge label $f *$ is defined as follows:

$$
\begin{aligned}
& f *\left(u_{1} v_{j}\right)=j \text { for } 1 \leq j \leq n, f *\left(u_{1} u_{2}\right)=\left\lceil\frac{2 n+1}{2}\right\rceil=n+1, \\
& f *\left(u_{m} v_{j}\right)=\left\lceil\frac{2 n+1+2 j}{2}\right\rceil=n+1+j \text { for } 1 \leq j \leq n \\
& f *\left(u_{i} u_{i+1}\right)=\left\lceil\frac{2 n+2 i-3+2 n+2 i-1}{2}\right\rceil=2 n+2 i-2 \text { for } 2 \leq i \leq\left\lceil\frac{m+1}{2}\right\rceil,
\end{aligned}
$$




$$
\begin{aligned}
& f *\left(u_{i} u_{i+1}\right)=\left\lceil\frac{2 n+2+2(m-i)+2 n+2+2(m-i-1)}{2}\right\rceil=2 n+2(m-i)+1 \text { for } \\
& \left\lceil\frac{m+1}{2}\right\rceil+1 \leq i \leq m-1 . \text { Now }\{f *(e): e \in E(G)\}=\{1,2,3, \ldots, m+2 n-1\} .
\end{aligned}
$$

It can be verified that $f$ is a mean labeling of $G$. Hence $G$ is a mean graph.

Example 2.11: The mean labeling of $P_{9}(+) \overline{K_{5}}$ is given in Fig. 5 .

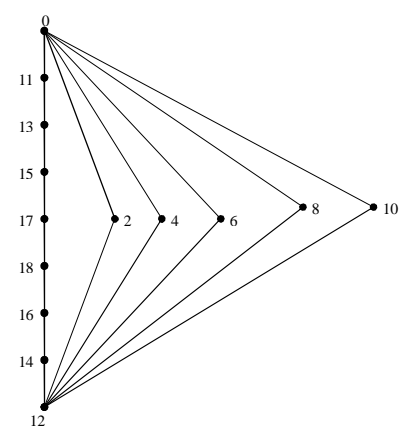

Fig. 5

Theorem 2.12: The graph Jelly fish $(J F)_{n}$ is a mean graph.

Proof: Let $V\left((J F)_{n}\right)=\left\{u, v, u_{i}, v_{j}: 1 \leq i \leq n, 1 \leq j \leq n-2\right\}$ and $E\left((J F)_{n}\right)=\left\{u u_{i}: 1 \leq i \leq n\right\} \cup\left\{v v_{j}: 1 \leq j \leq n-2\right\} \cup\left\{u_{1} u_{n}, v u_{1} v u_{n}\right\}$.

Define $f: V\left((J F)_{n}\right) \rightarrow\{0,1,2, \ldots, 2 n+1\}$ as follows:

$f(u)=0 ; f\left(u_{i}\right)=2 i$ for $1 \leq i \leq n ; f(v)=2 n+1 ; f\left(v_{j}\right)=2 j+3$ for $1 \leq j \leq n-2$.

For each vertex label $f$, the induced edge label $f *$ is defined as follows:

$$
\begin{aligned}
& f *\left(u u_{i}\right)=i \text { for } 1 \leq i \leq n, f *\left(v v_{j}\right)=n+j+2 \text { for } 1 \leq j \leq n-2 \\
& f^{*}\left(u_{1} u_{n}\right)=\left\lceil\frac{2 n+2}{2}\right\rceil=n+1, f *\left(v u_{1}\right)=\left\lceil\frac{2 n+3}{2}\right\rceil=n+2, f *\left(v u_{n}\right)=\left\lceil\frac{4 n+1}{2}\right\rceil=2 n+1 .
\end{aligned}
$$

Therefore, $\{f *(e): e \in E(G)\}=\{1,2,3, \ldots, n, n+1, n+2, \ldots, 2 n, 2 n+1\}$.

It can be verified that $f$ is a mean labeling of $(J F)_{n}$ and hence $(J F)_{n}$ is a mean graph.

Example 2.13: The mean labeling of $(J F)_{5}$ is given in Fig. 6. 


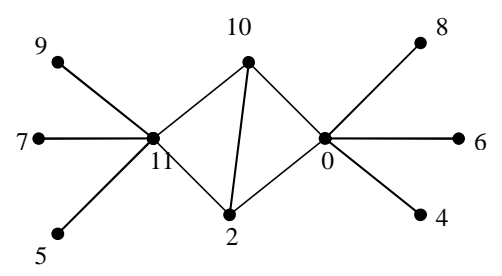

Fig. 6

Theorem 2.14: Let $G$ be a mean tree with $V(G)=\left\{v_{1}, v_{2}, \ldots, v_{p}\right\}$ and let $G^{\prime}$ be a copy of $G$ and with $V\left(G^{\prime}\right)=\left\{v_{1}{ }^{\prime}, v_{2}{ }^{\prime}, \ldots, v_{p}{ }^{\prime}\right\}$. Then the graph $G^{(+)}$obtained by joining the vertex $v_{i}$ with $v_{i}{ }^{\prime}$ by an edge for all $1 \leq i \leq p$, is a mean graph.

Proof: Let $f$ be a mean labeling of $G$. Clearly $V\left(G^{(+)}\right)=V(G) \cup V\left(G^{\prime}\right)$. Add the number $2 p-1$ to the label of the vertices $v_{i}{ }^{\prime}$ for $1 \leq i \leq p$. Then the vertex labels of the graph $G^{\prime}$ remain distinct and the edge labels of $G^{\prime}$ are increased by $2 p-1$. Since $G$ is a tree, $f(V(G))=\{0,1,2,3, \ldots, p-1\}$ and the edge labels of $G$ are $1,2,3, \ldots, p-1$. Also the induced edge labels of $G^{\prime}$ are $2 p, 2 p+1,2 p+2, \ldots, 3 p-2$. For each $i=1$ to $n$, the label of the edge $v_{i} v_{i}{ }^{\prime}$ is $\left[\frac{f\left(v_{i}\right)+f\left(v_{i}\right)+2 p-1}{2}\right\rceil=f\left(v_{i}\right)+p$. Therefore the induced edge labels of $v_{i} v_{i}{ }^{\prime}$ for $1 \leq i \leq p$ are $p, p+1, p+2, \ldots, 2 p-1$. Thus $G^{(+)}$is a mean graph.

Example 2.15: Let $G$ be a Comb obtained from the path $P_{4}$. The mean labeling of $G^{(+)}$is given in Fig. 7.

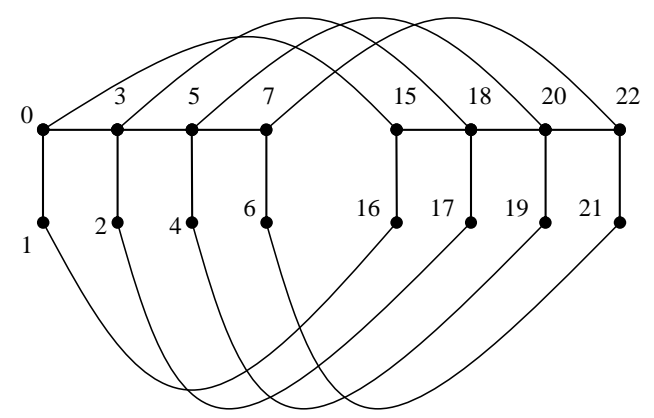

Fig. 7

Theorem 2.16: The graph $K_{n}{ }^{c}+2 P_{3}$ is a mean graph for all $\mathrm{n}$. 
Proof: Let $V\left(K_{n}\right)=\left\{u_{1}, u_{2}, u_{3}, \ldots, u_{n}\right\}$. Let $V\left(2 P_{3}\right)=\{u, v, w, x, y, z\}$ and $E\left(2 P_{3}\right)=\{u v, v w, x y, y z\}$.

Define $f: V\left(K_{n}{ }^{c}+2 P_{3}\right) \rightarrow\{0,1,2, \ldots, q=6 n+4\}$ as follows:

$f(u)=2, \quad f(v)=0$;

$f(w)=4, \quad f\left(u_{i}\right)=5+6(i-1)$ for $1 \leq i \leq n$,

$f(x)=6 n+1, f(y)=6 n+4, f(z)=6 n+3$.

For each vertex label $f$, the induced edge label $f *$ is defined as follows:

$$
\begin{array}{ll}
f *(u v)=1, & f *(v w)=2, \\
f^{*}\left(u u_{i}\right)=3 i+1 & \text { for } 1 \leq i \leq n, \\
f^{*}\left(v u_{i}\right)=3 i & \text { for } 1 \leq i \leq n, \\
f^{*}\left(w u_{i}\right)=3 i+2 & \text { for } 1 \leq i \leq n, \\
f^{*}\left(x u_{i}\right)=3(n+i) & \text { for } 1 \leq i \leq n, \\
f^{*}\left(y u_{i}\right)=3(n+i)+2 & \text { for } 1 \leq i \leq n, \\
f^{*}\left(z u_{i}\right)=3(n+i)+1 & \text { for } 1 \leq i \leq n, \\
f^{*}(x y)=6 n+3, & f *(y z)=6 n+4 .
\end{array}
$$

It can be verified that $f$ is a mean labeling and hence $K_{n}{ }^{c}+2 P_{3}$ is a mean graph.

Example 2.17: The mean labeling of $K_{3}{ }^{c}+2 P_{3}$ is given in Fig. 8 .

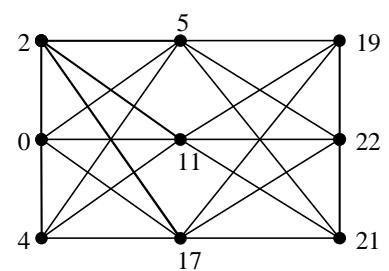

Fig. 8

\section{References}

1. F. Harary, Graph theory (Addison Wesley, Massachusetts, 1972). PMid:4261464

2. S. Somasundaram and R. Ponraj, National Acad. Sci. Lett. 26, 210 (2003).

3. S. Somasundaram and R. Ponraj, Pure Appl. Mathematika Sci. 58, 29 (2003).

4. D. Ramya and P. Jeyanthi, SUT J. Math. 47 (2), 129 (2011).

5. P. Jeyanthi, D. Ramya, and A. Maheswari, Utilitas Mathematica 98, 2014 (In press). 